\title{
Neutral and Nonneutral Mutations: The Creative Mix-Evolution of Complexity in Gene Interaction Systems
}

\author{
Emile Zuckerkandl \\ Institute of Molecular Medical Sciences, 460 Page Mill Road, Palo Alto, CA 94306, USA
}

Re: J Mol Evol (1997) 44 (Suppl 1):S2-S8. Page S4, 2nd column, line 1: Instead of " . . . the full activity of the duplicate gene would not be limited to the embryo" read "... the full activity of the the duplicate gene would now be limited to the embryo." 